\title{
Large scale deletions in the GPC3 gene may account for a minority of cases of Simpson-Golabi-Behmel syndrome
}

Susan Lindsay, Maggie Ireland, Ottie O'Brien, Jill Clayton-Smith, Jane A Hurst, Jillian Mann, Trevor Cole, Julian Sampson, Sarah Slaney, David Schlessinger, John Burn, Giuseppe Pilia

Department of Human Genetics, Ridley Building, University of Newcastle upon Tyne, Claremont Place, Newcastle upon Tyne NE1 7RU, UK

S Lindsay

$M$ Ireland

O O'Brien

J Burn

Department of Clinical Genetics, St Mary's

Hospital, Manchester,

UK

J Clayton-Smith

Department of Clinical Genetics, Churchill

Hospital, Oxford, UK

J A Hurst

Department of Oncology, The Children's Hospital, Birmingham, UK J Mann

Clinical Genetics Unit, Birmingham Women's Hospital,

Birmingham, UK

T Cole

Institute of Medical Genetics, University Hospital of Wales, Cardiff, UK

J Sampson

Institute of Child Health, London, UK S Slaney

Center for Genetics in Medicine, Washington University School of Medicine, St Louis, USA

D Schlessinger

Istituto di Clinica e Biologia dell'Eta Evolutiva, Ospedale Microcitemie, Cagliari, Italy G Pilia

Correspondence to: Dr Lindsay.

Received 28 November 1996 Revised version accepted for publication 10 January 1997

\begin{abstract}
Aims of the study-To identify the proportion and type of deletions present in the glypican 3 (GPC3) gene in a group of patients with Simpson-Golabi-Behmel syndrome (SGBS).
\end{abstract}

Subjects and methods-PCR analysis using primer pairs which amplify fragments from each of the eight exons of the GPC3 gene was carried out in a series of 18 families with SGBS (approximately half of reported cases).

Results-Deletions were detected in only five families (one reported previously). We found deletions in all exons of the gene except exon 3.

Conclusions-Our results suggest that large scale deletions may be less common in SGBS than was originally thought. One patient, with an exon 4 and 5 deletion, lacked the characteristic facial dysmorphic features. This raises the possibility of involvement of GPC3 gene defects in a wider range of overgrowth disorders. (F Med Genet 1997;34:480-483)

Keywords: Simpson-Golabi-Behmel syndrome; glypican 3; X linked; overgrowth disorder

Simpson-Golabi-Behmel syndrome (SGBS) is an $\mathrm{X}$ linked overgrowth syndrome associated with characteristic facial features that are usually described as being coarse in quality. Although early motor milestones and speech are delayed, most affected males are of normal intelligence. Abnormalities of the skeletal system, heart, central nervous system, kidney, and gastrointestinal tract may also be present. ${ }^{1-4}$ In addition, some affected subjects develop embryonal tumours. ${ }^{5}$ Recently, the gene responsible for SGBS has been cloned and shown to be glypican 3 (GPC3), ${ }^{6}$ a member of the glypican family of heparin sulphate proteoglycans. The expression pattern of GPC3 suggests that it may play an important role in growth control in embryonic mesoderm and endoderm derived tissues. Initial ligand and western blotting experiments have suggested that GPC3 may interact with insulinlike growth factor 2 (IGF2), which may be significant given the overlap in phenotype between Beckwith-Wiedemann syndrome (BWS) and SGBS patients.
The known sequence of the GPC3 transcript is $2130 \mathrm{bp}$ and spans approximately $500 \mathrm{~kb}$ of genomic DNA. Initial studies, examining six of the eight exons, identified deletions in three of the six patients tested. ${ }^{6}$ This suggested that large scale deletions might be responsible for a significant proportion of cases of SGBS. This would not be unexpected given the large region of genomic DNA covered by the GPC3 gene and the high proportion of deletions found in some other disorders involving other large genes (for example, in the dystrophin gene in patients with Duchenne muscular dystrophy)

Aims of the study

The aims of the study were to identify the proportion and type of deletions present in the eight exons of the GPC3 gene in SGBS patients from 18 families.

\section{Patients and methods}

PATIENTS

Blood samples were collected from patients and their families and DNA prepared following standard methods. Table 1 shows the main clinical features in the 18 index cases studied. Family 7 was reported as family $C$ in reference 6 . Of the 18 cases, five are familial (SGBS-1, 5, 7,19 , and 20) and 13 appear to be sporadic cases. With the exception of SGBS-2, 9, 19, and 20 , all the cases were ascertained by contacting clinical geneticists and paediatric oncologists in the UK. The patient and his family in SGBS-2 are from Angola (of Portuguese ancestry), while those in SGBS-9 are from Germany, ${ }^{8}$ SGBS-19 and $20^{3}$ from Austria, and SGBS-17 from The Netherlands The patient in SGBS-4 is Afro-Caribbean and those in SGBS-5 are of Lithuanian-Italian extraction.

The characteristic facial features of SGBS and their development with age are illustrated in fig 1 which shows a male from family SGBS- 1 at birth, 3 years, and 7 years of age The characteristic facial features, which are al present at 7 years of age, include a broad nose prominent jaw, upward slanting eyes, longitudinal groove in the tongue, and central groove in the lower lip. Note that the face appears to develop with age; at birth the face looks relatively normal, but by 3 years the nose is broad and the eyes upward slanting but the jaw is still of normal size. In contrast, one of the patients in SGBS-5 is shown in fig 2. In this 

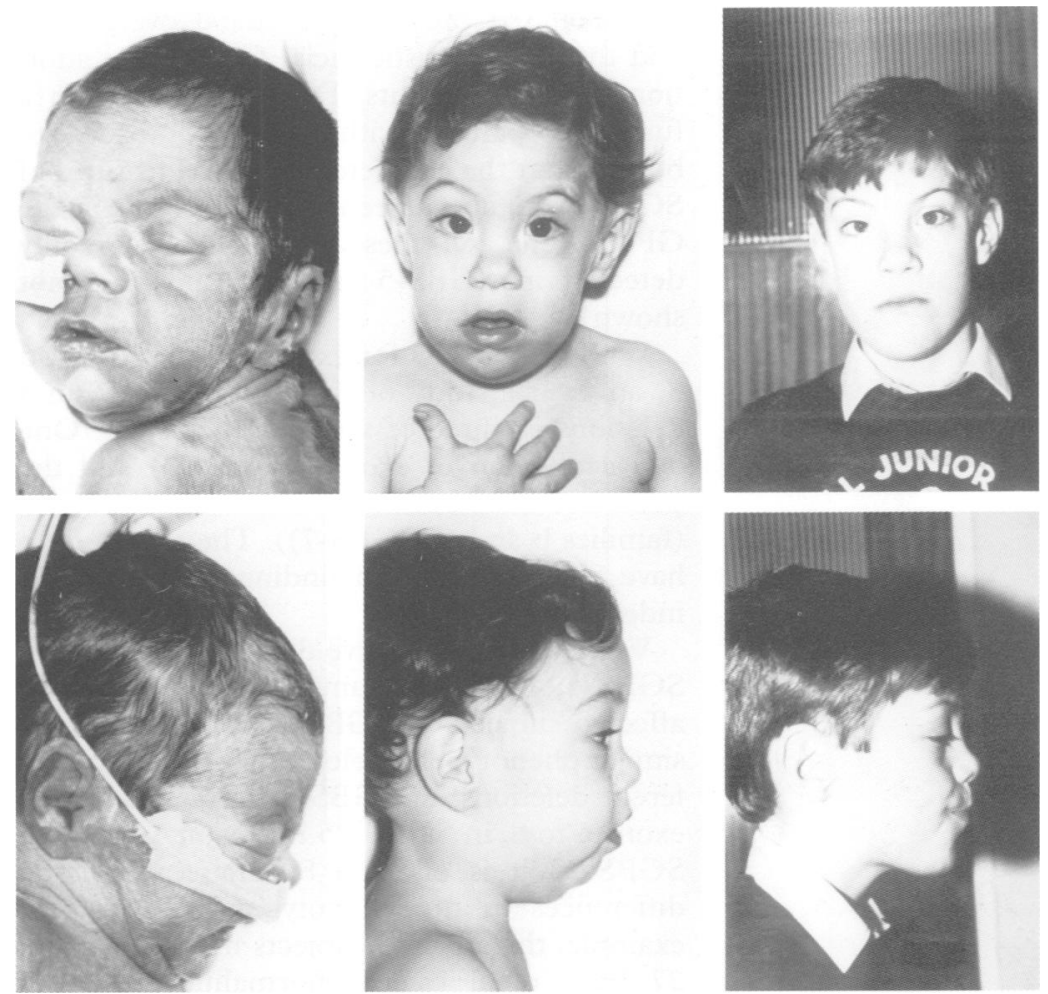

Figure 1 One of the affected males in family SGBS-1 at birth, 3 years, and 7 years of age. See text for further discussion. (All photographs reproduced with permission.)

family the facial features are less striking, in particular the broad midface is absent (see below for further discussion).

\section{Methods}

PCR was carried out using the six sets of primer pairs previously defined. ${ }^{6}$ These are intraexonic primers for exons $1,2,3,5,6$, and 8. Primers for exons 4 and 7 have since been defined and are as follows: exon 4 (forward) $5^{\prime}$ gcttattatcctgaagatctc 3'; (reverse) 5' ttctttggaataggtcgg 3'; exon 7 (forward) 5' tgagaaccatgtctatgc 3'; (reverse) 5' cggtgatgatgaagatga 3'.

For each exon, one primer was end labelled with $\gamma^{32} \mathrm{P}$ ATP using T4 polynucleotide kinase (MBI Fermentas) using the manufacturer's buffer and protocol. PCR was carried out in reaction volumes of $10 \mu \mathrm{l}$ containing standard manufacturer's buffer, $200 \mu \mathrm{mol} / 1 \mathrm{dNTPs}, 0.5$ $\mu \mathrm{mol} / 1$ forward and reverse primers, $100 \mathrm{ng}$ of genomic DNA, and 1 unit of $\mathrm{TBr}$ polymerase

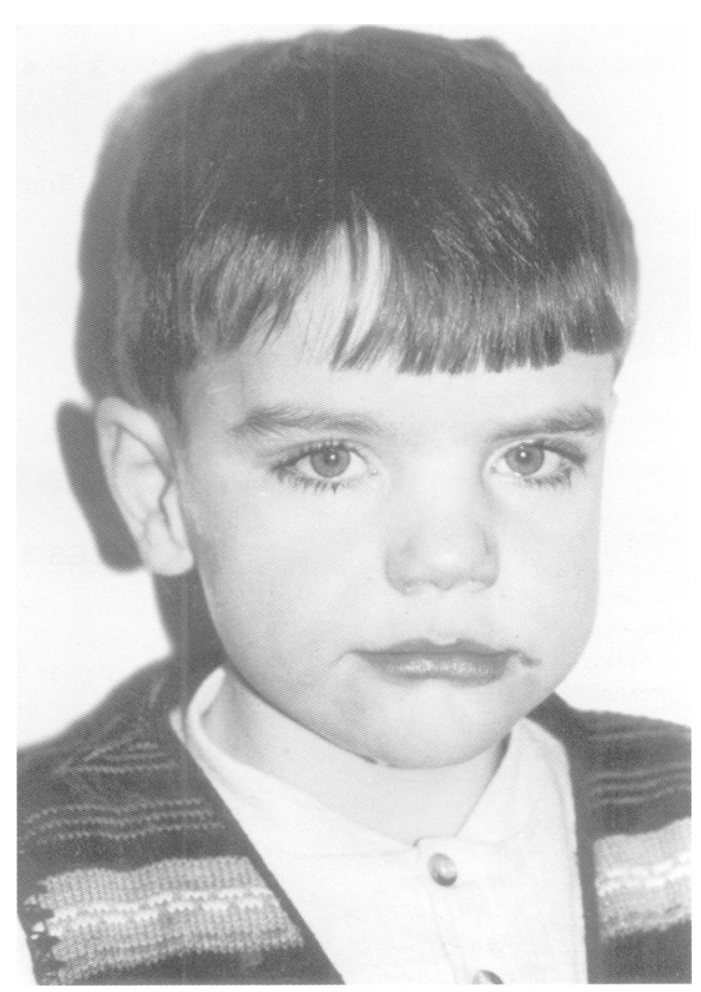

Figure 2 One of the affected males in SGBS-5. See text for further discussion.

(NBL). The reaction cycles were as follows: $94^{\circ} \mathrm{C}$ for one minute, annealing temperature for one minute, $72^{\circ} \mathrm{C}$ for two minutes for 32 cycles. This was preceded by a three minute incubation at $94^{\circ} \mathrm{C}$ and followed by a 10 minute incubation at $72^{\circ} \mathrm{C}$. The annealing temperatures were exon $160^{\circ} \mathrm{C}$, exon $253^{\circ} \mathrm{C}$, exons $3,4,5$, and $755^{\circ} \mathrm{C}$, exons 6 and $857^{\circ} \mathrm{C}$. The reaction products were electrophoresed on denaturing acrylamide gels which were dried down and exposed to XAR5 autoradiographic film at $-80^{\circ} \mathrm{C}$ overnight.

\section{Results}

A summary of the deletions identified is given in table 2, while fig 3 shows representative results obtained for exons 2,6 , and 8 . From table 2 it can be seen that deletions were detected in exons 1 and 2 in SGBS-4, in exons 4 and 5 in SGBS-5, in exons 6,7 , and 8 in SGBS-7, in exon 1 in SGB-25, and in exon 8 in

Table 1 Clinical features of patients with SGBS

\begin{tabular}{|c|c|c|c|c|c|c|c|c|c|c|c|c|c|c|c|c|c|c|}
\hline & \multicolumn{18}{|c|}{ SGB Family No } \\
\hline & 1 & 2 & 3 & 4 & 5 & 6 & 7 & 8 & 9 & 10 & 11 & 12 & 16 & 17 & 19 & 20 & 25 & 27 \\
\hline Prenatal overgrowth & + & + & + & + & + & + & + & + & + & + & + & + & + & + & - & + & + & + \\
\hline Postnatal overgrowth & + & + & + & + & + & + & + & + & + & + & + & + & + & + & + & + & + & + \\
\hline $\begin{array}{l}\text { Delayed speech } \\
\text { Delayed motor }\end{array}$ & + & + & + & + & + & + & + & + & - & + & - & + & & + & + & + & + & + \\
\hline milestones & + & + & + & + & + & + & + & + & - & + & + & + & & + & + & + & + & + \\
\hline CNS abnormalities & - & - & - & - & - & - & + & - & - & - & - & + & - & - & - & - & - & - \\
\hline $\begin{array}{l}\text { Cardiac malformation } \\
\text { Characteristic facial }\end{array}$ & + & - & - & - & + & - & + & - & + & - & + & + & - & - & + & + & - & - \\
\hline $\begin{array}{l}\text { features } \\
\text { Renal tract }\end{array}$ & + & + & + & + & $+1-$ & + & + & + & + & + & + & + & + & + & + & + & + & + \\
\hline abnormalities & + & + & - & + & + & - & + & - & - & + & - & - & - & - & - & - & - & + \\
\hline $\begin{array}{l}\text { Embryonal tumours } \\
\text { Supernumerary }\end{array}$ & - & + & - & + & - & - & - & - & - & - & - & - & - & - & - & - & - & - \\
\hline nipples & + & + & - & - & + & + & + & + & + & + & + & + & - & - & - & - & - & - \\
\hline Skeletal abnormalities & + & + & - & - & + & - & - & + & + & + & + & - & - & - & - & + & $+/-$ & - \\
\hline Hernias & - & - & - & + & - & + & - & + & - & + & - & - & - & - & + & + & + & - \\
\hline
\end{tabular}



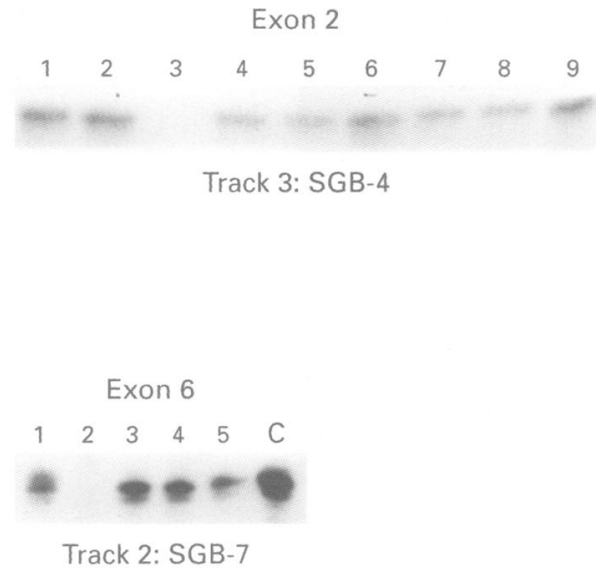

C

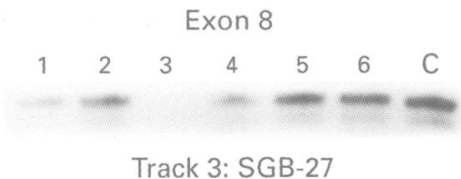

Figure 3 Examples of the deletion analysis in SGBS patients. (A) Amplification of exon 2. Track 3 contains $D N A$ from the affected subject in $S G B S-4$. (B)

Amplification of exon 6. Track 2 contains $D N A$ from the affected subject in SGBS-7. (C) Amplification of exon 8. Track 3 contains DNA from the affected subject in $S G B S-27$. Other tracks contain PCR fragments amplified from other SGBS families or from a GPC3 $c D N A$ clone (labelled C) as a control. All reactions have been carried out at least twice. In all DNA in which a deletion was detected other exons were amplified without problem.

SGBS-27. SGBS-4, 25, and 27 are isolated cases while there are two affected subjects in each of SGBS-5 and SGBS-7. Deletions of exons 6 and 8 had already been reported in SGBS-7. ${ }^{6}$ This study shows that exon 7 is also deleted in both affected subjects in the family (table 2 and data not shown). The deletion of exons 4 and 5 was present in both affected subjects in SGBS-5 (data not shown). No deletions were detected in any of the other cases, which includes SGBS-20 the family originally reported by Professor Behmel and her colleagues. ${ }^{3}$

\section{Discussion}

Five deletions have been found in 18 SGBS patients screened with exonic primer sets for all eight exons of the GPC3 gene. Seventeen of these patients (all but SGBS-5) were clearly identified as having SGBS because their phenotypes were characteristic of this disorder and included pre- and postnatal overgrowth and the characteristic facial features. In addition to these patients, DNA samples from a further 13 patients with overgrowth disorders, but without the characteristic facial features of SGBS, were also screened for deletions in the GPC3 gene. Of these, a deletion was only detected in SGBS-5 (table 2 and data not shown).

With primer sets for exons $1,2,3,5,6$, and 8 , an earlier study of the GPC3 gene found deletions in three of six patients tested. ${ }^{6}$ One had a deletion of exon 2 (family a) and the other two were both deleted for exons 6 and 8 (families b and c (SGBS-7)). The same group have recently reported finding a further four independent deletions. ${ }^{9}$

We have identified five different deletions in SGBS-4, 5, 7, 25, and 27. Although the affected subjects in SGBS-7, 25, and 27 have similar phenotypes (table 1) they have very different deletions: in SGBS-7 the deletion is of exons 6 to 8, in SGBS-25 it is exon 1, while in SGBS-27 it is of exon 8 . There are subtle differences in the phenotypes, however; for example, the affected subjects in SGBS-7 and 27 have renal tract abnormalities while in SGBS-25 the affected person does not. In addition to the features shown in table 1 , SGBS-25 also had a submucous cleft palate which was repaired. The younger of the two affected males in SGBS-7 developed hydrocephalus and has required the insertion of a ventriculoperitoneal shunt. This complication has not previously been described in SGBS, although a male in SGBS-12 is similarly affected. The significance of these differences is unclear at present.

The affected male in SGBS-4 (deleted for exons 1 and 2) had originally been diagnosed as having BWS. He was ascertained in a follow up study of UK cases of BWS. His facial features, which included a broad midface, upward slanting palpebral fissures, grooved tongue, and notch in the lower lip, are much more characteristic of SGBS. ${ }^{4}$ In contrast to most SGBS patients, however, he had moderate/mild psychomotor retardation. At the age of 7 he had a Wilms' tumour removed but despite chemotherapy he died from a recurrence. Affected subjects in a previously reported family with a deletion of exon $2^{6}$ also had a number of embryonal tumours but were of normal intelligence. ${ }^{5}$ The affected subject in SGBS-25, who is also of normal intelligence, is deleted for exon 1. SGBS-4 is deleted for both exons 1 and 2 and, in contrast, has significant

Table 2 Results of deletion screening in 18 European families

\begin{tabular}{|c|c|c|c|c|c|c|c|c|c|c|c|c|c|c|c|c|c|c|}
\hline \multirow{2}{*}{$\begin{array}{l}\text { Exon } \\
\text { No }\end{array}$} & \multicolumn{18}{|c|}{$S G B$ family $N o$} \\
\hline & 1 & 2 & 3 & 4 & 5 & 6 & 7 & 8 & 9 & 10 & 11 & 12 & 16 & 17 & 19 & 20 & 25 & 27 \\
\hline 1 & + & + & + & - & + & + & + & + & + & + & + & + & + & + & + & + & - & + \\
\hline 2 & + & + & + & - & + & + & + & + & + & + & + & + & + & + & + & + & + & + \\
\hline 3 & + & + & + & + & + & + & + & + & + & + & + & + & + & + & + & + & + & + \\
\hline 4 & + & + & + & + & - & + & + & + & + & + & + & + & + & + & + & + & + & + \\
\hline 5 & + & + & + & + & - & + & + & + & + & + & + & + & + & + & + & + & + & + \\
\hline 6 & + & + & + & + & + & + & - & + & + & + & + & + & + & + & + & + & + & + \\
\hline 7 & + & + & + & + & + & + & - & + & + & + & + & + & + & + & + & + & + & + \\
\hline 8 & + & + & + & + & + & + & - & + & + & + & + & + & + & + & + & + & + & - \\
\hline
\end{tabular}


psychomotor retardation. The deletion in this case may extend 5 ' to the GPC3 gene to involve other genes. To investigate this possibility, the extent of the deletion in the patient in SGBS-4 is currently being characterised. In addition, the results from this study and that of HughesBenzie et al suggest that mutations in the 5 end of the gene may be involved in tumour development in SGBS patients. The affected subject in SGBS-2, however, also had a Wilms' tumour (table 1) and no deletion was detected in exons 1 or 2 (or any other exon) (table 2). However, it remains to be seen whether there is another type of mutation in either of these exons in this family and also what the true frequency of embryonal tumours is in SGBS.

The facial features of the two males in SGB-5 are not typical of SGBS (fig 2). They lack the broad midface but the older of the two has a rather prominent jaw and upward slanting palpebral fissures. However, there were pre- and postnatal overgrowth, delayed motor milestones, and other features suggestive of SGBS (table 1). Interestingly, in this case exons 4 and 5 were deleted and this was not seen in any of the more classical SGBS cases. It may be advisable, therefore, to extend the analysis of the GPC3 gene to patients with preand postnatal overgrowth who have abnormalities in other organ systems, but who lack the characteristic facial features of SGBS.

There is significant overlap in phenotype between SGBS and BWS. ${ }^{10}$ Indeed, this and the fact that SGBS has only relatively recently been delineated as a single entity probably means that SGBS is underdiagnosed. The IGF2 gene (which maps to $11 \mathrm{p} 15.5$ ) has been implicated in the underlying molecular pathology of BWS ${ }^{7}$ and there is now evidence to suggest that there is an interaction between GPC3 and IGF $2{ }^{6}$ Evidence from mouse mutants also indicates that abnormalities of IGF2 expres- sion affect growth. ${ }^{11} 12$ It may, therefore, also be valuable to look for mutations in the GPC3 gene in male BWS patients, for whom there is no indication of chromosome 11 involvement.

We are grateful to the following clinical geneticists and paediatric oncologists for supplying us with samples from patients and their families: Dr Annemarie Behmel, Dr Louise Brueton, D Brian Coppin, Professor Alan Craft, Dr Nick Dennis, Dr Amanda Collins, Dr Heather Fletcher, Dr Judith Goodship, D Eli Hatchwell, Dr Raoul Hennekam, Shirley Hodgson, D Rainer Konig, Alison Lashwood, Dr Ana Medeira, Dr Ann Slavotinek, Dr Sabine Stengel-Rutkovsky, and Professor Ian Young. We would also like to thank Mr Charles Stiller and the Childhood Cancer Research Group (Oxford). In addition, SL would like to acknowledge the support of the Wellcome Trus would JB, and MI thank the North East Children's Cance and SL, JB, and MI thank the North East Children's Cance Italian Telethon (award No E.357) for research support.

1 Simpson JL, Landey S, New M, German JA. A previously unrecognised X-linked syndrome of dysmorphia. Birth Defects 1975; XI:18-24.

2 Golabi M, Rosen L. A new X-linked mental retardation overgrowth syndrome? Am $\mathcal{F}$ Med Genet 1984;17:345-58.

3 Behmel A, Plochi E, Rosenkranz W. A new X-linked dysplasia gigantism syndrome: identical with the Simpson dysplasia syndrome? Hum Genet 1984;67:409-13.

4 Neri G, Marini R, Cappa M, Borelli P, Opitz JM. SimpsonGolabi-Behmel syndrome: an X-linked encephalotrophoschisis syndrome. Am f Med Genet 1988;30:287-99.

5 Hughes-Benzie RM, Hunter AG, Allanson JE, Mackenzi AE. Simpson-Golabi-Behmel syndrome associated with renal dysplasia and embryonal tumours: localisation of the gene to Xqcen-q21. Am $\mathcal{F}$ Med Genet 1994;43:428-35.

6 Pilia G, Hughes-Benzie RM, Mackenzie AE, et al. Mutations in GPC3, a glypican gene, cause the SimpsonGolabi-Behmel overgrowth syndrome. Nat Genet 1996;12 241-7.

7 Weksberg R, Squire JA, Templeton DM. Glypicans: a growing trend. Nat Genet 1996;12:225-7.

8 Konig R, Fuchs S, Kern C, Langenbeck U. SimpsonGolabi-Behmel syndrome with severe cardiac arrhythmias. Am $\mathcal{F}$ Med Genet 1991;38:244-7.

9 Hughes-Benzie RM, Xuan JY, Pilia G, Schlessinger D Mackenzie AE. Simpson-Golabi-Behmel syndrome: mutation analysis of the causative GPC gene and preliminary biochemical assessment of GPC. Am $\mathcal{f}$ Hum Gene 1996;59:A140.

10 Hughes-Benzie RM, Allanson J, Hunter A, Cole T. The importance of differentiating Simpson-Golabi-Behmel and Beckwith Wiedemann syndromes. 7 Med Genet 1992;29. 928.

11 De Chiara TM, Robertson EJ, Efstratiadis A. Parental imprinting of the mouse insulin-like growth factor 2 gene. Cell 1991;64:849-59.

12 Wang ZQ, Fung MR, Barlow DP, Wagner EF. Regulation of embryonic growth and lysosomal targeting by the imembryonic growth and lysosomal targeting by
printed IGF2/MPR gene. Nature 1994;372:464-7. 